\title{
A finite-volume/level-set interface capturing method for unstructured grids: simulations of bubbles rising through viscous liquids
}

\author{
N. Balcázar ${ }^{1}$, L. Jofre $^{1}$, O. Lehmkuhl ${ }^{1,2}$, J. Rigola $^{1}$, J. Castro $^{1}$ \\ \& A. Oliva ${ }^{1}$ \\ ${ }^{1}$ Heat and Mass Transfer Technological Center (CTTC), Universitat \\ Politècnica de Catalunya - BarcelonaTech (UPC) ETSEIAT, Spain \\ ${ }^{2}$ Termo Fluids, S.L., Spain
}

\begin{abstract}
A numerical method is developed for simulating two-phase flows with moving inter-phase boundaries, which is integrated in a finite-volume framework on collocated unstructured grids of arbitrary element type. The location, geometry and the movement of the discontinuities are described by the conservative levelset method. The proposed algorithm is validated with experimental results of the buoyant rise of an isolated bubble. Then the method is applied to simulate the interaction between two bubbles during their buoyant rise. Finally, simulations for a set of bubbles with the same diameter initially placed in a random pattern in a periodic cylindrical duct are also carried out. In general, a good agreement is found between the current simulations and results reported in the literature.

Keywords: finite-volume method, conservative level set method, incompressible two phase flow, unstructured grid.
\end{abstract}

\section{Introduction}

The level-set method, which was first derived by Osher and Sethian (1988) [2], is a versatile method for capturing the motion of free surfaces. In this approach the interface is defined implicitly by the zero contour of a signed distance function. The evolution of this function in space and time is governed by an advection equation, combined with a special redistancing algorithm. An advantage 
of the level-set algorithm is its simplicity to compute geometric properties of the interface, required for instance to calculate the curvature of the surface. A disadvantage of this method, is that the discrete solution of transport equations is prone to numerical error and leads to loss or gain of mass. Recently Olsson and Kreiss [1] proposed a conservative level set (CLS) method, where mass conservation problem that is known to affect the LS method is greatly reduced. Additionally this approach benefits of automatic handling of topology changes and efficient parallelization with no additional cost.

The main objective of this paper is to propose a numerical method to solve incompressible two-phase flows with moving inter-phase boundaries on unstructured meshes. The location, geometry and the movement of the interface is described by the CLS method of Olsson and Kreiss [1]. The governing equations are discretized by the finite-volume formulation on a collocated grid arrangement. A projection method is used to solve incompressible Navier-Stokes equations that are coupled to a transport equation for the level set function. The effect of surface tension is modeled by using the continuum surface force approach (CSF) according to Brackbill et al. [5].

The proposed algorithm is applied to compute the terminal shapes and Reynolds number of three-dimensional gas bubbles rising in stagnant liquids for low to high Eotvos number and Morton number. The rise and shape of two bubbles rising in a stagnant liquid and the evolution of the bubble interaction process are examined. Finally, a set of bubbles rising in a periodic duct is simulated.

The outline of the paper is as follows: A summary of the governing equations is given in section 2. Section 3 discusses the numerical method. Code validation and numerical results are presented in section 4 . The conclusion is given in section 5 .

\section{Governing equations}

\subsection{Conservative level set equations}

Instead of the signed distance function $d(\mathbf{x}, t)$ used to represent the interface in the classical LS method, Olsson and Kreiss [1] employed a regularized indicator function $\phi(\mathbf{x}, t)=(1+\exp (d(\mathbf{x}, t) / \varepsilon))^{-1}$ in the context of their CLS method. This function takes the value 0 in one fluid and the value 1 in the other fluid, whereas the interface is defined by the location of the $\phi=0.5$ iso-surface. The interface transport equation can be written in conservative form provided the velocity field is solenoidal:

$$
\frac{\partial \phi}{\partial t}+\nabla \cdot \phi \mathbf{v}=0
$$

Furthermore, an additional re-initialization equation is introduced to keep the profile and thickness of the interface constant,

$$
\frac{\partial \phi}{\partial \tau}+\nabla \cdot \phi(1-\phi) \mathbf{n}_{\tau=0}=\nabla \cdot \varepsilon \nabla \phi
$$


This equation is advanced in pseudo-time $\tau$, it consists of a compressive flux $\phi(1-\phi) \mathbf{n}_{\tau=0}$ that aims at sharpening the profile, and of a diffusion term $\nabla \cdot \varepsilon \nabla \phi$ that ensure the profile remains of characteristic thickness $\varepsilon$. Geometrical information on the interface, such as normal vector $\mathbf{n}$ or curvature $\kappa$, is obtained through:

$$
\mathbf{n}=\frac{\nabla \phi}{\|\nabla \phi\|} \quad \kappa(\phi)=-\nabla \cdot \mathbf{n}
$$

\subsection{Incompressible two-phase flow}

The conservation of momentum and mass of two immiscible incompressible fluids is described by the Navier-Stokes equations defined on a spatial-time domain $\Omega \times[0, T]$ with boundary $\partial \Omega$ :

$$
\begin{aligned}
& \left\{\begin{array}{l}
\frac{\partial}{\partial t}(\rho \mathbf{v})+\nabla \cdot(\rho \mathbf{v v})=-\nabla p+\nabla \cdot \mu\left(\nabla \mathbf{v}+(\nabla \mathbf{v})^{T}\right)+\rho \mathbf{g}+\sigma \kappa \nabla \phi \\
\beta=\beta_{1} \phi+\beta_{2}(1-\phi) \quad \text { with } \beta \in\{\rho, \mu\} .
\end{array}\right. \\
& \nabla \cdot \mathbf{v}=0
\end{aligned}
$$

where $\rho$ and $\mu$ denote the density and dynamic viscosity of the fluids, $\mathbf{v}$ is the velocity field, $\mathbf{g}$ is the gravity acceleration, $p$ is the pressure, and $\sigma$ is the coefficient of surface tension.

\section{Numerical method}

In this work, the finite-volume (FV) method is used to discretize the governing equations on a collocated grid arrangement. The presented numerical methods are implemented in an in-house solver called TermoFluids [4].

\subsection{Spatial discretization}

The spatial discretization is performed by using the integral form of the governing equations over the control volume $P$ :

$$
\int_{V_{P}} \frac{\partial \xi \psi}{\partial t} d V_{P}=\oint_{A_{P}}\left(-\xi G^{\mathbf{c}}(\psi) \mathbf{c}+\lambda \nabla \psi\right) \cdot d \mathbf{A}_{P}+\int_{V_{P}} S_{\psi} d V_{P}
$$

Here, $\psi \in\left\{v_{x}, v_{y}, v_{z}, \phi\right\}, \xi \in\{\rho, 1\}, \mathbf{c} \in\{\mathbf{v}, \mathbf{n}\}, S_{\psi}$ is the source term, $\mathbf{A}_{f}$ is the area vector of the cell face $f, V_{P}$ is the volume of the cell $P$ and $A_{P}$ is the surface of the cell $P$. In what follows, next definitions will be used: $\psi_{P} \equiv \psi\left(\mathbf{x}_{P}\right)=\frac{1}{V_{P}} \int_{V_{P}} \psi d V_{P}$ and $\psi_{f} \equiv \psi\left(\mathbf{x}_{f}\right)=\frac{1}{A_{f}} \int_{A_{f}} \psi d A_{f}$. 
Table 1: General conservation Eq. (6). Source term in Eq. (4) is expressed in cartesian tensor notation, where $x_{i} \in\{x, y, z\}, v_{i} \in\left\{v_{x}, v_{y}, v_{z}\right\}$, $\partial_{x_{i}} \equiv \partial / \partial x_{i}$ and $g_{i} \in\left\{g_{x}, g_{y}, g_{z}\right\}$.

\begin{tabular}{lcccccc}
\hline Equation & $\psi$ & $\xi$ & $G^{\mathbf{c}}(\psi)$ & $\mathbf{c}$ & $\lambda$ & $S_{\psi}$ \\
\hline Momentum Eq.(4) & $v_{i}$ & $\rho$ & $v_{i}$ & $\mathbf{v}$ & $\mu$ & $-\partial_{x_{i}} p+\partial_{x_{j}} \mu \partial_{x_{i}} v_{j}+\rho g_{i}$ \\
& & & & & & $+\sigma \kappa \partial_{x_{i}} \phi$ \\
Advection CLS Eq.(1) & $\phi$ & 1 & $\phi$ & $\mathbf{v}$ & 0 & 0 \\
Re-initialization CLS Eq.(2) & $\phi$ & 1 & $\phi(1-\phi)$ & $\mathbf{n}$ & $\varepsilon$ & 0 \\
\hline
\end{tabular}

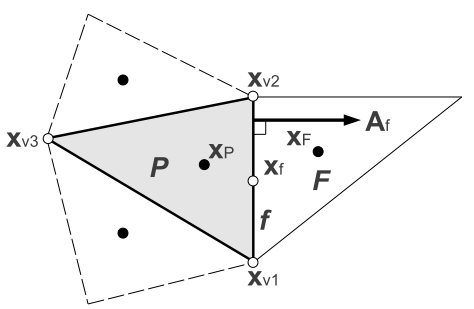

$(\mathbf{a})$
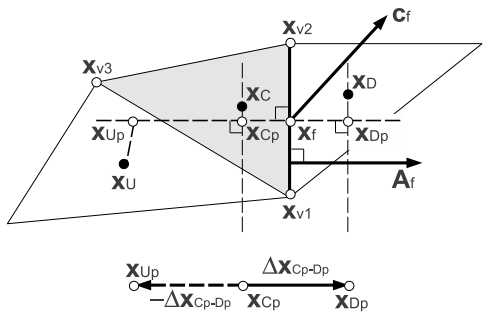

(b)

Figure 1: Nomenclature used in spatial discretization: (a) Source term and diffusive node stencil. (b) Convective and compressive node stencil.

\subsubsection{Face interpolation and gradient reconstruction}

All variables are defined at cell centroids in a collocated grid arrangement. Hence, a distance-weighted linear interpolation is used to find the face values:

$$
\xi_{f}=\sum_{q \in\{P, F\}} w_{q} \xi_{q}, \text { where } w_{q}=\frac{\left\|\Delta \mathbf{x}_{f \rightarrow q}\right\|}{\sum_{q \in\{P, F\}}\left\|\Delta \mathbf{x}_{f \rightarrow q}\right\|}
$$

The application of a truncated Taylor series $\psi_{F i}=\psi_{P}+\left(\mathbf{x}_{F i}-\mathbf{x}_{P}\right) \cdot(\nabla \psi)_{P}$ to all points included in a cell stencil $\mathrm{i}=1,2, \ldots, n$, where $i$ is a neighbor cell of $P$, gives a system of linear equations for the derivatives at centroid $\mathbf{x}_{P}$. The resulting over-determined system of linear equations $\mathbf{M}(\nabla \psi)_{P}=\mathbf{Y}$ is solved by the leastsquares method: $(\nabla \psi)_{P}=\left(\mathbf{M}^{T} \mathbf{M}\right)^{-1} \mathbf{M}^{T} \mathbf{Y}$.

\subsubsection{Diffusive term}

The diffusive term is discretized by using a central difference scheme:

$$
\oint_{A_{P}} \lambda \nabla \psi \cdot d \mathbf{A}_{P} \approx \sum_{f} \lambda_{f}\left\|\mathbf{A}_{f}\right\|\left(\frac{\psi_{F}-\psi_{P}}{\left\|\Delta \mathbf{x}_{P \rightarrow F}\right\|}+\frac{(\nabla \psi)_{f} \cdot \Delta \mathbf{x}_{v 1 \rightarrow v 2}}{\left\|\Delta \mathbf{x}_{v 1 \rightarrow v 2}\right\|}\right)
$$




\subsubsection{Convective and compressive terms}

The convective and compressive terms are approximated as follows:

$$
\begin{aligned}
\oint_{A_{c v}} \xi G^{\mathbf{c}}(\psi) \mathbf{c} \cdot d \mathbf{A}_{P} & \approx \sum_{f}\left[\left[\xi_{f} U_{f}^{\mathbf{c}}, 0\right]\right] G\left(\psi_{C_{p}}\right)-\left[\left[-\xi_{f} U_{f}^{\mathbf{c}}, 0\right]\right] G\left(\psi_{D_{p}}\right) \\
& +\frac{1}{2} \xi_{f} U_{f}^{\mathbf{c}} L\left(\theta_{f}\right)\left(G\left(\psi_{D_{p}}\right)-G\left(\psi_{C_{p}}\right)\right)
\end{aligned}
$$

where, $\mathbf{c} \in\{\mathbf{v}, \mathbf{n}\}, U_{f}^{\mathbf{v}} \equiv \mathbf{v}_{f} \cdot \mathbf{A}_{f}$ is the volume flux, $U_{f}^{\mathbf{n}} \equiv \mathbf{n}_{f} \cdot \mathbf{A}_{f}$ is the compression flux, $L(\theta)$ is the flux limiter function, $\theta_{f}=\left(\psi_{C_{p}}-\right.$ $\left.\psi_{U_{p}}\right) /\left(\psi_{D_{p}}-\psi_{C_{p}}\right)$ is the upwind ratio of consecutive gradients of the solution; where $\psi_{C_{p}}, \psi_{U_{p}}, \psi_{D_{p}}$ are calculated by a truncated Taylor series, for instance $\psi_{C_{p}}=\psi_{C}+\left(\mathbf{x}_{C_{p}}-\mathbf{x}_{C}\right) \cdot(\nabla \psi)_{C}$. The operator $[[a, b]]$ denotes the maximum of the two arguments $a$ and $b, \xi_{f}$ and $U_{f}^{\mathbf{n}}$ are calculated by linear interpolation, whereas $U_{f}^{\mathbf{v}}$ is obtained by using a mass-conservative interpolation scheme. To avoid unphysical oscillations in the level set function, a TVD Superbee limiter, $L(\theta)=\max \{0, \min \{2 \theta, 1\}, \min \{2, \theta\}\}$, is used to discretize the convective term in advection Eq. (1). Central difference limiter, $L(\theta)=1$, is used to discretize the convective term and compressive term of momentum Eq. (4) and re-initialization Eq. (2) respectively.

\subsubsection{Source term}

The source term in the momentum equations (see Table 1) is discretized as follows:

$$
\int_{V_{P}} S_{\mathbf{v}} d V_{P} \approx \sum_{f}\left(-p_{f} \mathbf{A}_{f}+\mu_{f}(\nabla \mathbf{v})_{f}^{T} \cdot \mathbf{A}_{f}\right)+\mathbf{f}_{P}
$$

where, $\mathbf{f}_{P}=\rho_{P} \mathbf{g} V_{P}+\sigma \kappa_{P}(\nabla \phi)_{P} V_{P}$. The source term $S_{\phi}$ in advection Eq. (1) and re-initialization Eq. (2) is zero. Face values are evaluated by linear interpolation and gradients are computed by the least-squares method.

\subsubsection{Interface thickness, normal and curvature}

Solving Eq. (2) to steady-state results in a smooth transition of $\phi$ at the interface that depends of the diffusion coefficient $\varepsilon_{P}=C_{\varepsilon}\left(\Delta_{P}\right)^{\alpha}$, where $\Delta_{P}=\left(V_{P}\right)^{1 / 3}$. In numerical experiments we have found that the value $C_{\varepsilon}=0.5$ and $0.9 \leqslant \alpha \leqslant 1$, are satisfactory.

The interface normal vector $\mathbf{n}_{P}=(\nabla \phi)_{P} /\left\|(\nabla \phi)_{P}\right\|$ is calculated by the leastsquares method. The curvature is directly computed from the interface normal field $\kappa_{P}=\frac{1}{V_{P}} \sum_{f} \mathbf{n}_{f} \cdot \mathbf{A}_{f}$, where $\mathbf{n}_{f}$ is evaluated by linear interpolation.

\subsection{Time discretization}

Both the advection Eq. (1) and re-initialization Eq. (2) are integrated in time with a 3-step third-order accurate TVD Runge-Kutta scheme [3]. A stability restriction due to the viscous term is applied to solve Eq. (2). Thus, the pseudo-time step $\Delta \tau$ 
is taken as $\Delta \tau=C_{\tau}\left(\min \left\{\Delta_{P}\right\}\right)^{2} / \varepsilon$, where $C_{\tau} \approx 0.01$. Only a few steps are needed to reach the steady state of Eq. (2).

The Navier-Stokes equations are integrated by using a sequence of fractional steps [10]. First, an intermediate velocity field is obtained using an explicit Adams-Bashforth scheme:

$$
\mathbf{v}_{P}^{*}=\mathbf{v}_{P}^{n}+\frac{\Delta t}{\rho\left(\phi_{P}^{n}\right) V_{P}}\left(\frac{3}{2}\left(\mathbf{R}_{\mathbf{v}}\right)_{P}^{n}-\frac{1}{2}\left(\mathbf{R}_{\mathbf{v}}\right)_{P}^{n-1}\right)
$$

where $\left(\mathbf{R}_{\mathbf{v}}\right)_{P}$ is the spatial discretization of convective, diffusive and source terms in momentum Eq. (4), excluding the pressure term. In step two, the velocities at the new time instant $t_{n+1}$ are computed by:

$$
\mathbf{v}_{P}^{n+1}=\mathbf{v}_{P}^{*}-\frac{\Delta t}{\rho\left(\phi_{P}^{n}\right)}\left(\nabla p^{n+1}\right)_{P}
$$

Under the incompressibility constraint of Eq. (5), divergence of Eq. (12) leads to

$$
\sum_{f} \mathbf{v}_{f}^{*} \cdot \mathbf{A}_{f}=\sum_{f}\left(p_{F}^{n+1}-p_{P}^{n+1}\right) \frac{\Delta t\left\|\mathbf{A}_{f}\right\|}{\rho_{f}\left\|\Delta \mathbf{x}_{P \rightarrow F}\right\|}
$$

This is a variable coefficient Poisson equation for the pressure, which is solved using a preconditioned conjugate gradient technique. To avoid pressure-velocity decoupling when the pressure projection is made on collocated meshes [11], a cell face volume flux is defined so that fluxes conserve mass exactly:

$$
\begin{aligned}
\left(U_{f}^{\mathbf{v}}\right)^{n+1} & =\sum_{q \in\{P, F\}} w_{q}\left(\mathbf{v}_{q}^{n+1}+\frac{\Delta t}{\rho\left(\phi_{q}^{n}\right)}\left(\nabla p^{n+1}\right)_{q}\right) \cdot \mathbf{A}_{f} \\
& -\frac{\Delta t}{\rho_{f}}\left(\nabla p^{n+1}\right)_{f} \cdot \mathbf{A}_{f}
\end{aligned}
$$

In order to minimize the kinetic energy error, linear interpolation in Eqs. (14) is performed by using mesh-independent weights of $w_{q}=0.5$ (Felten [11]).

\subsection{Solution algorithm}

For each time update the numerical scheme is summarised as follows:

1. Choose an appropriate time step $\Delta t$.

2. Advance the conservative level set function $\phi$ solving Eq. (1).

3. The re-initialization Eq. (2) is solved for steady state.

4. Curvature, density and viscosity fields are updated from the level set field.

5. The Navier-Stokes equations are solved by the fractional-step method, through the solution of Eq. (11), Eq. (13) and Eq. (12).

6. Update the mass conservative velocity $U_{f}$ solving Eq. (14).

7. Repeat step 1 to step 6 until the desired time-level is reached. 


\section{Numerical experiments}

\subsection{Single bubble rise}

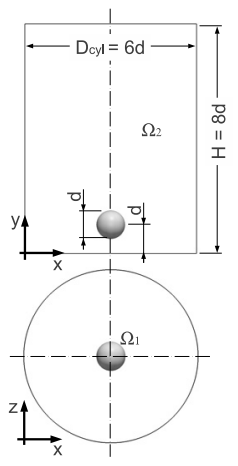

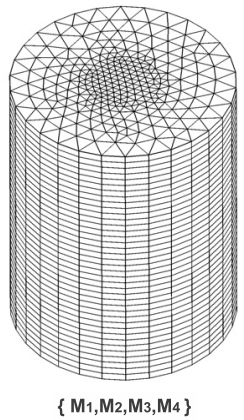

(a)
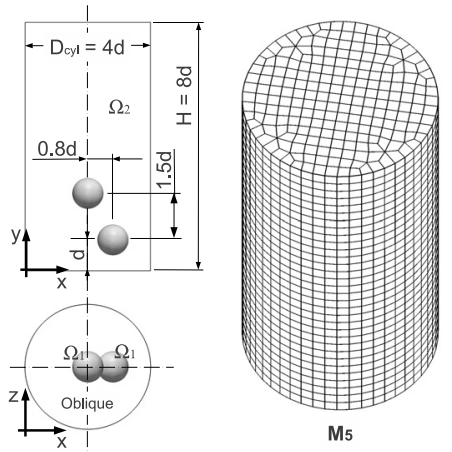

(b)

Figure 2: The schematic diagram of the solution domain and mesh configuration. (a) Single bubble rise: The mesh is densified around the symmetry axis of the cylinder $\left(M_{1}: 7.6 \times 10^{5} \mathrm{cv} \equiv 15 \mathrm{cv} / \mathrm{d}, M_{2}: 1.4 \times 10^{6} \mathrm{cv} \equiv\right.$ $\left.20 \mathrm{cv} / d, M_{3}: 2.3 \times 10^{6} \mathrm{cv} \equiv 25 \mathrm{cv} / d, M_{4}: 3.4 \times 10^{6} \mathrm{cv} \equiv 30 \mathrm{cv} / d\right)$. (b) Bubble merge: The mesh is uniformly distributed in the cylinder $\left(M_{5}: 1.4 \times 10^{6} \mathrm{cv} \equiv 25 \mathrm{cv} / d\right)$.
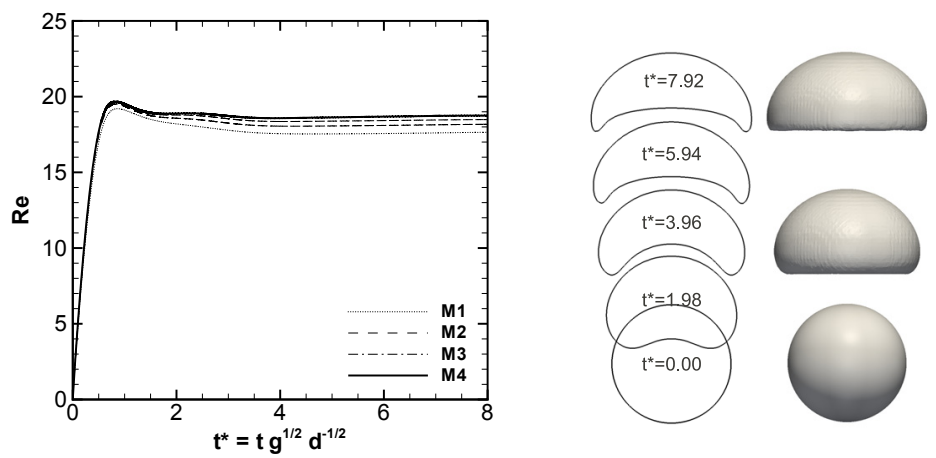

Figure 3: Single bubble rise: $E o=97.1, M o=0.971, \rho_{1} / \rho_{2}=100, \mu_{1} / \mu_{2}=$ 100. 
To validate the numerical method, the buoyant rise of a single bubble is first simulated. For bubbles rising freely in infinite media a generalized graphical correlation is given by Grace [6]. This diagram shows the shape regimes and terminal velocities in terms of Eötvös number $E_{O}=g d^{2} \Delta \rho / \sigma$, Morton number $M=g \mu_{1}^{4} \Delta \rho /\left(\rho_{1}^{2} \sigma^{3}\right)$ and Reynolds number $R e=\rho_{1} U_{T} d / \mu_{1}$. Here, $U_{T}$ is used to denote the terminal velocity of the bubble, $\Delta \rho=\rho_{1}-\rho_{2}$ specifies the density difference between the fluid phases, the subscript 1 refers to the heavier fluid and the subscript 2 to the lighter fluid.

Computational domain and mesh configuration are described in Fig. 2a. According to Mukundakrishnan et al. [12], when $D_{c y l} \geq 6 d$, the effect of sidewalls is minimal. Height of the cylinder tube is taken $H=8 d$. Initially the spherical bubble of diameter $d$ is located on the symmetric axis at $d$ from the bottom wall. Both liquid and bubble are assumed initially quiescent. No-slip boundary conditions are applied on top, side and bottom walls.

The density and viscosity ratios are specified respectively as $\rho_{1} / \rho_{2}=100$ and $\mu_{1} / \mu_{2}=100$. Numerical studies have been performed in the past using these parameters by [8]. The time step size is fixed to $\Delta t^{*}=2.0 \times 10^{-4} \mathrm{~s}$ except for cases $A, B$ and $C$ (see Table 4) where the time step is reduced to $\Delta t^{*}=5 \times 10^{-5}$. All computations were made using an interface thickness parameter of $\varepsilon=0.5\left(\Delta_{P}\right)^{0.9}$.

Numerical results for various values of the Eo number and the $M$ number are shown in Fig. 4. The dimensionless numbers reported here are evaluated at the initial stage using the droplet diameter $d$. From the simulations presented in Fig. 4, it can be concluded the current numerical method is robust enough to predict the various bubble shapes under a wide range of flow regimes. The comparison of the terminal Reynolds numbers for the experiment and simulation cases is listed in Table 2 . The results from simulations agree with those of experiments very well.

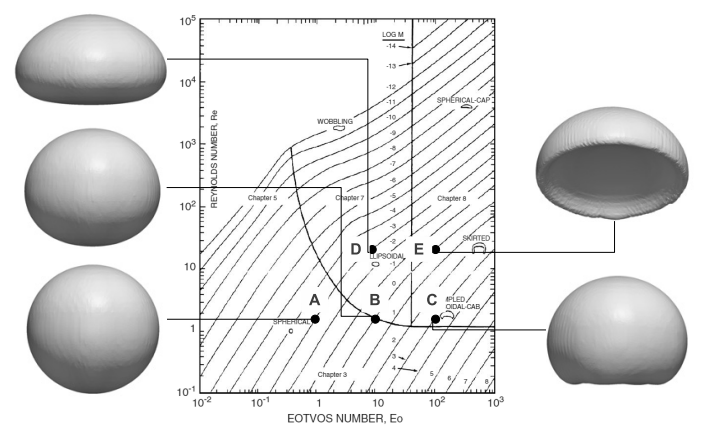

Figure 4: Bubble diagram of Grace for the shape and terminal velocities of gas bubbles in quiescent viscous liquids, reproduced from Clift and Weber [7]. The simulated cases are indicated with capitals. 
Table 2: Simulation of rising bubble for different regimes according to the bubble diagram of Grace [6]. $R e_{G}$ represents the experimental Reynolds number obtained from the Grace diagram and $R e_{C}$ represents the predicted Reynolds number.

\begin{tabular}{lllllll}
\hline Bubble regime & $M$ & $E o$ & $R e_{G}$ & $R e_{C}$ & Case & Mesh \\
\hline Spherical & $1.26 \times 10^{-3}$ & 1.0 & 1.7 & 1.68 & $\mathbf{A}$ & $M_{3}$ \\
Ellipsoidal & $1.00 \times 10^{0}$ & 10.0 & 1.7 & 1.75 & $\mathbf{B}$ & $M_{3}$ \\
Ellipsoidal Cap. & $1.00 \times 10^{3}$ & 97.1 & 1.5 & 1.58 & $\mathbf{C}$ & $M_{3}$ \\
Ellipsoidal & $9.71 \times 10^{-4}$ & 10.0 & 22.0 & 23.0 & $\mathbf{D}$ & $M_{3}$ \\
Skirted & $9.71 \times 10^{-1}$ & 97.1 & 20.0 & 18.7 & $\mathbf{E}$ & $M_{4}$ \\
\hline
\end{tabular}

\subsection{Bubble merge}

The oblique coalescence of two rising bubbles in an initially quiescent liquid is explored. The initial bubble arrange and mesh configuration are shown in the Fig. 2b. The simulation results are performed using $\varepsilon=0.5\left(\Delta_{c v}\right)^{0.9}$ and time step $\Delta t^{*}=2.0 \times 10^{-4}$. The fluid parameters are: $E_{o}=16, M=2.0 \times 10^{-4}$, $\rho_{1} / \rho_{2}=100, \mu_{1} / \mu_{2}=100$, where subscripts 2 and 1 refer to the outer-liquid phase and bubble phase respectively. Free-slip boundary condition is used at lateral side of the domain and no-slip boundary condition at the top and bottom boundaries.

A sequence of shapes for coalescence process of two bubbles is presented in Fig. 5. Bubbles start to rise due to buoyancy, subsequently, a vortex is created in the wake of the upper bubble. The suction by the top bubble produce the collision between them and the thin liquid film between the bubbles is squeezed out and ruptured. Finally, the two bubbles merge into a single bubble, completing the coalescence process. The numerical prediction match fairly well in terms of bubble shapes with the experimental results reported by Brereton and Korotney [9]. The similar observations have been found by van Sint Annaland et al. [8] using volumeof-fluid method (VOF) [8].

\subsection{Bubbly flow}

In this section, a set of 30 rising bubbles with the same diameter $d$ is simulated in a cylindrical duct, for $\rho_{1} / \rho_{2}=10, \mu_{1} / \mu_{2}=10, E o=2.25$ and $M=6 \times 10^{-4}$. Bubbles are initially placed in the domain in a random pattern. The volume fraction occupied by bubbles in the whole domain is $0.034 \%$, and the minimal distance between the bubbles at initial state is $1.25 d$. The domain size and mesh configuration are the same as in the previous case (see Fig. 2b). But, in this case the domain is discretized by $12.6 \times 10^{6} \mathrm{cv} \equiv 30 \mathrm{cv} / \mathrm{d}$. The simulation results are performed using $\varepsilon=0.5\left(\Delta_{c v}\right)^{0.9}$ and time step $\Delta t^{*}=2.0 \times 10^{-4}$. The periodic 


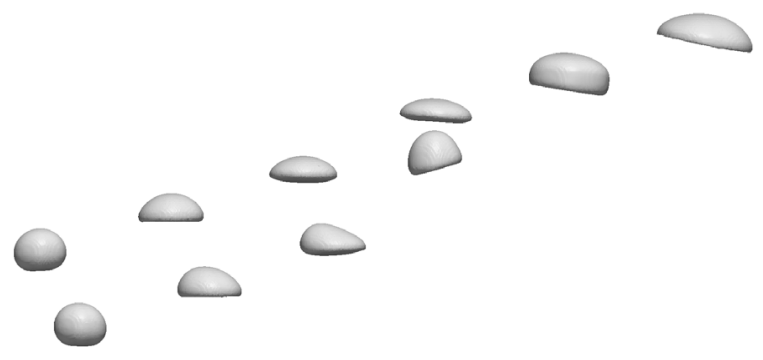

Figure 5: Oblique bubble coalescence of two initially spherical bubbles. Here $M=0.0002$, Eo $=16, \rho_{1} / \rho_{2}=100, \mu_{1} / \mu_{2}=100$.

boundary condition is applied at the top and bottom of the domain, whereas no-slip boundary condition is applied at the side wall.

Fig. 6 shows the pattern of bubbly flow. Bubbles rise due the buoyancy force, and form its own wake. The dynamic interaction process of bubbles is dominated by wake effects. A bubble moving in the wake of another bubble is attracted toward the leading bubble. The strength of influence depends of distance between bubbles. Coalescence processes are observed as the time is increased, and large bubbles are formed, which then induces a stronger flow field that affect the neighbor bubbles. The bubbles in this regime flow moves through the center of the cylindrical duct, which indicates that bubbles near the symmetry axis are moving faster.

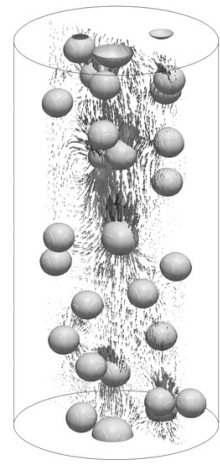

$t^{*}=11.2$
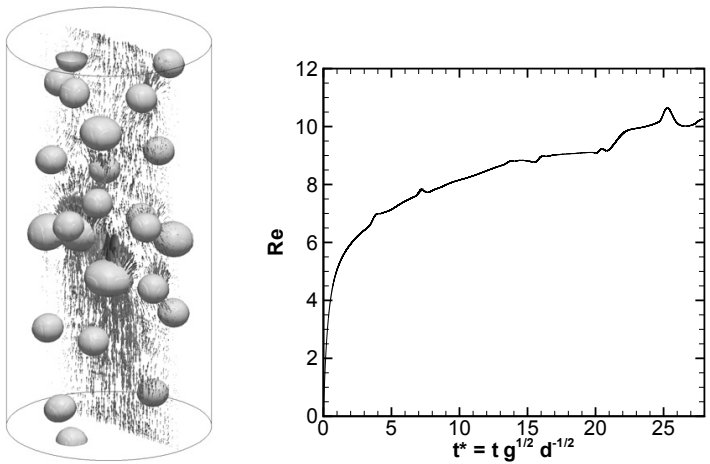

$t^{*}=14.0$

Figure 6: Bubbly flow: $M=6.0 \times 10^{-4}, E o=2.25, \rho_{1} / \rho_{2}=10, \mu_{1} / \mu_{2}=10$. 


\section{Conclusions}

In this work, a numerical method for simulating incompressible two-phase flows on unstructured grids was presented. This method was based on the CLS technique, using a collocated FV scheme to discretize the governing equations. Such a combination of the numerical techniques results in a fast and efficient algorithm that allows to simulate virtually all kinds of two-phase flows with inter-phase boundaries (bubbles, waves, films etc.). Numerical simulations of bubbles rising freely in quiescent liquids were performed for different flow regimes. In general, results are in good agreement with numerical and experimental results of the literature. It is found that the present method is a viable tool for accurate modeling of two-phase flows on unstructured grids.

\section{Acknowledgements}

This work has been supported by the Ministerio de Economía y Competitividad (ENE-2011-28699), Spain. The authors wish to thank the Barcelona Supercomputing Center for the use of computational facilities. Nestor Balcazar acknowledge financial support of the Agencia Española de Cooperación Internacional para el Desarrollo (AECID), Spain.

\section{References}

[1] Olsson E., Kreiss G., A conservative level set method for two phase flow, $J$. Comput. Phys., 210, pp. 225-246, 2005.

[2] Osher S., Sethian J.A., Fronts propagating with curvature-dependent speed: Algorithms based on Hamilton-Jacobi formulations, J. Comput. Phys., 79, pp. 175-210, 1998.

[3] Gottlieb S., Chi-Wang S., Total Variation Dimishing Runge-Kutta Schemes, Mathematics of Computations, 67, pp. 73-85, 1998.

[4] Lehmkuhl O., Perez-Segarra C.D., Soria M., Oliva A., 2007, A new Parallel unstructured CFD code for the simulation of turbulent industrial problems on low cost PC cluster, Proceedings of the Parallel CFD 2007 Conference, pp. 1-8, 2007.

[5] Brackbill J., kothe B., Zemach C., A Continuum Method for Modeling Surface Tension, J. Comput. Phys., 100, pp. 335-354, 1992.

[6] Grace J.R., Shapes and velocities of bubbles rising in infinite liquids, Trans IChemE, 51, pp. 116-120, 1973.

[7] R. Clift, M. Weber, Bubbles, Drops and Particles, Academic Press, New York, 1978.

[8] M. van Sint Annaland, N.G. Deen, J.A.M. Kuipers, Numerical Simulation of gas bubbles behaviour using a three-dimensional volume-of-fluid method, Chemical Engineering Science, 60, pp. 1999-3011, 2005. 
[9] G. Brereton, D. Korotney, Coaxial and Oblique Coalescence of Two Rising Bubbles, in Dynamics of bubbles and Vortices Near a Free Surface, ASME 119, New York, 1991.

[10] J.L. Guermond, P. Minev, Jie Shen, An overview of projection methods for incompressible flows, Comput. Methods Appl. Mech. Engrg., 195, pp. 60116045, 2006.

[11] Frederic N. Felten, Thomas S. Lund, Kinetic energy conservation issues associated with the collocated mesh scheme for incompressible flow, J. Comput. Phys., 215, pp. 465-484, 2006.

[12] K. Mukundakrishnan, S. Quan, D.M. Eckmann, P.S. Ayyaswamy, Numerical study of wall effects on buoyant gas-bubble rise in a liquid-filled finite cylinder, Phys. Rev. E., 76, 036308-01-036308-15, 2007. 